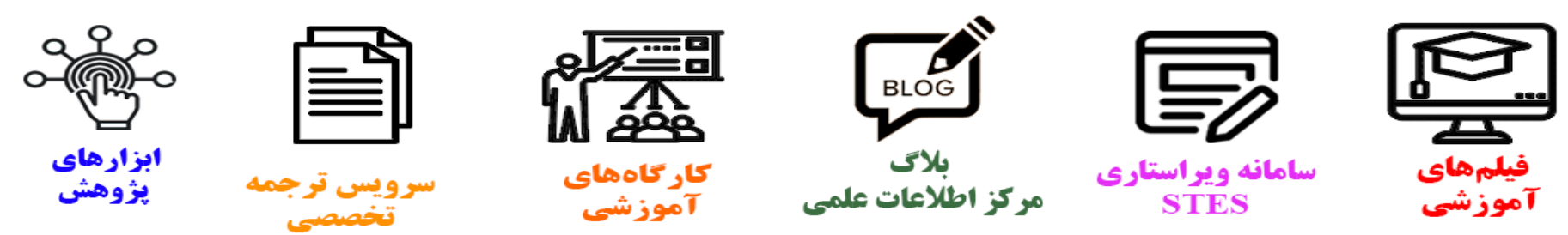

\title{
(c)
}

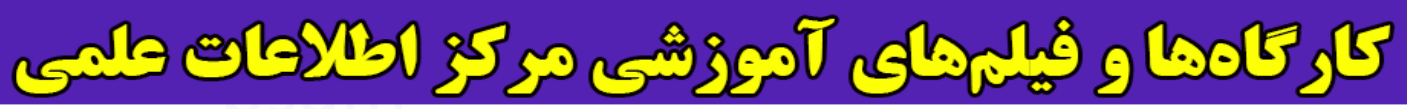
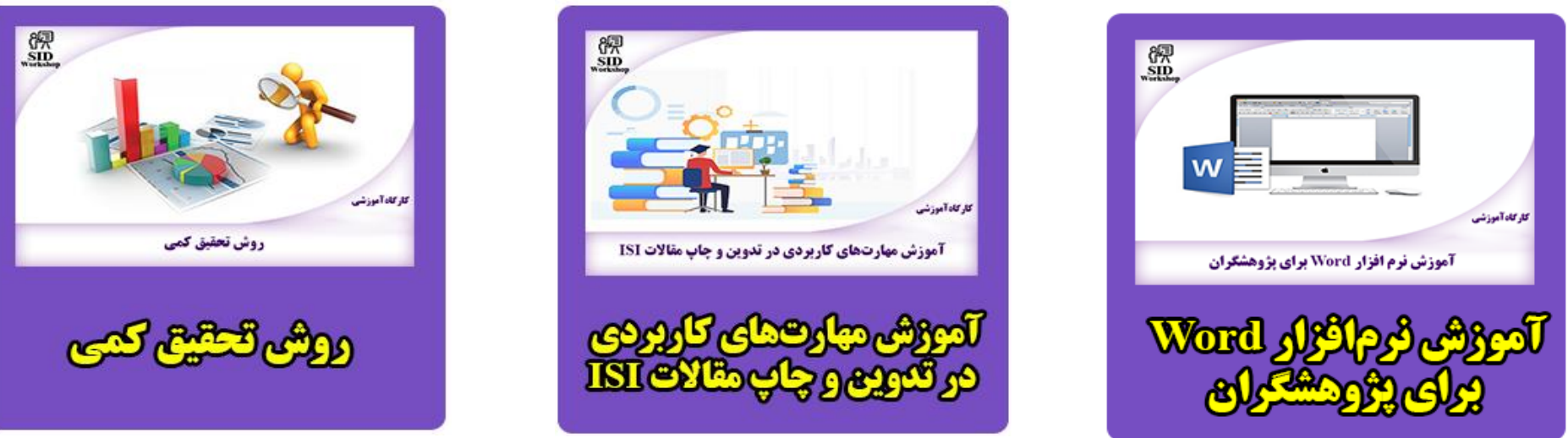


\title{
International Journal of Engineering
}

\section{Analytical Analysis of Capacitive Pressure Sensor with Clamped Diaphragm}

\author{
M. Shahiri Tabarestania, B. Azizollah Ganji *b \\ ${ }^{a}$ Department of Electrical Engineering, Islamic Azad University, Central Tehran Branch, Tehran, Iran \\ ${ }^{b}$ Electrical Engineering Department, Babol University of Technology, Babol, Iran
}

\section{PAPER INFO}

\section{Paper history:}

Received 24 May 2012

Received in revised form 23 July 2012

Accepted 15 Novemberr 2012

\section{Keywords:}

Capacitance

Deflection

Capacitive Pressure Sensor

Diaphragm Deflection

\section{A $B$ S S T R R A C C T}

In this paper, analytical analysis of capacitive pressure sensor with clamped diaphragm is presented. Mechanical and electrical properties of the sensor are theoretically analyzed based on theory of thin plates with small deflection and the results are evaluated using finite element analysis. The central deflection and capacitance values under uniform external pressure are calculated. Comparison of theoretical results shows good agreement with finite element analysis. The results indicate that the mathematical model has a high accuracy to determine the sensor behaviors.

\section{INTRODUCTION}

Capacitive micro machined pressure sensors have been designed for different kinds of applications because of high pressure sensitivity, low power consumption, and good stability compared to piezoresistive structures [13]. Generally, Micro-electromechanical system (MEMS) capacitive pressure sensor consists of a thin, flexible conductive membrane (diaphragm) as one of the electrodes that is separated from a fixed back plate by a small air gap. Due to an applied pressure, the displacement of the diaphragm is dependent on diaphragm's shape. There are two shapes of diaphragms (square and round) which are commonly applied in MEMS devices. In capacitive sensors, a thin diaphragm is used as a pressure sensing element [2-4]. When the diaphragm is exposed to an external uniform pressure, $\mathrm{P}$, the diaphragm deflects cause a decrease in the air gap that results an increase in capacitance between the diaphragm and the fixed back plate. So, deflection of the movable part due to applied pressure is sensed and translated into an electrical capacitance change [1].

The sensitivity of the capacitive sensors largely depends on the capacitance change, so exact calculation of the capacitance between the deformed diaphragm and the back plate is necessary. Parallel plate capacitor is the

*Corresponding Author Email: baganji@nit.ac.ir (B. Azizollah Ganji) basic and simplest form of capacitor. When one of the electrodes is deflected due to an external pressure, a parallel plate equation will introduce significant error. So, differential elements of parallel capacitance combined together to generate the total capacitance. Thus, it can be concluded that exact calculation of the capacitance depends on the physical position of all nodes on the diaphragm. Understanding of capacitance changes in the structure requires more knowledge about mechanical deflection of an upper electrode. The behaviors of the flat clamped diaphragms are investigated using the classical Timoshenko plate theory $[5,6]$. Many researchers have developed small and large deflection analysis for constant thickness plates [7].

In this paper, analytical analysis of capacitive pressure sensor with clamped diaphragm is presented. Furthermore, the mechanical deflection and capacitance changes of a capacitive pressure sensor are evaluated. Bending mechanics of rectangular thin plates with small deflection and with proper boundary conditions are calculated using mathematical software. To evaluate the analytical results, finite element analysis is used and compared with the analytical results. The paper is organized as follows; in section 2, the problem is illustrated using related equations. Finite element analysis of the pressure sensor is described in section 3 . The analytical and simulation results are summarized in section 4. Finally, section 5 concludes the paper. 


\section{PROBLEM ILLUSTRATION}

In pressure sensors, pressure is specified by the diaphragm deflection due to applied pressure. Figure 1 illustrates top and cross-section view of the typical pressure sensor diaphragm. The diaphragm side length and thickness are $a$ and $h$, respectively. $W_{c}$ denotes the center deflection and $w(x, y)$ is the deflection of the diaphragm. When the external pressure applied to the diaphragm, the diaphragm deflects. As the pressure is pulled back, the diaphragm returns to its original place. The boundary conditions of the clamped edges can be expressed mathematically as [6]:

$$
\begin{aligned}
& w(x= \pm a, \forall y)=0, \\
& w(y= \pm b, \forall x)=0, \\
& \frac{d w}{d x}(x= \pm a, \forall y)=0, \\
& \frac{d w}{d y}(y= \pm b, \forall x)=0 .
\end{aligned}
$$

The deflection, $w(x, y)$, caused by applying pressure to the uniform plate surface can be defined through the solution of this equation [6]:

$$
\frac{\partial^{4} w(x, y)}{\partial x^{4}}+2 \frac{\partial^{4} w(x, y)}{\partial x^{2} \partial y^{2}}+\frac{\partial^{4} w}{\partial y^{4}}=\frac{P(x, y)}{D}
$$

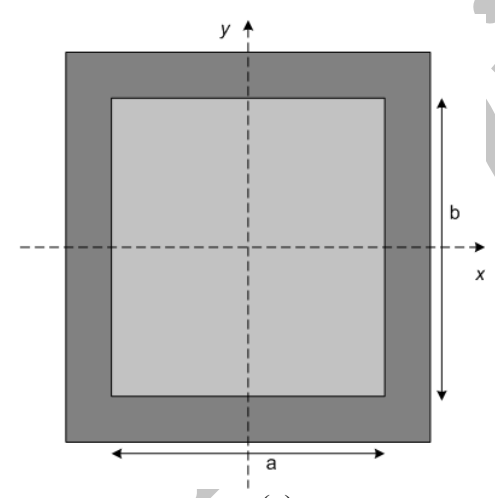

(a)

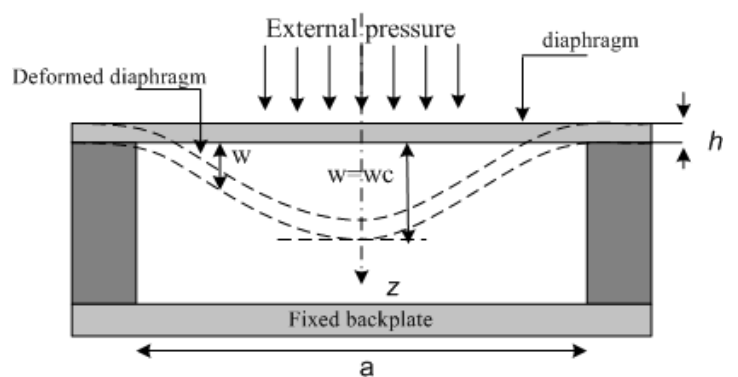

(b)

Figure 1. Top and cross section view of typical pressure sensor Diaphragm where, $P(x, y)$ is the applied pressure on the plate, and $D$ is called the flexural rigidity of the plate which is defined as:

$$
D=\frac{E h^{3}}{12\left(1-v^{2}\right)}
$$

where, $E$ is the modulus of elasticity of the material or the Young's modulus, $v$ is Poisson's ratio and $h$ is the thickness of the diaphragm. From [6] by applying clamped boundary conditions, the deflection $w(x, y)$, caused by applying pressure would be the sum of two components:

$$
W=W_{1}+w_{2}
$$

Where, $w_{1}$ is the deflection of the simply supported plate under uniform applied pressure and $W_{2}$ is representing the effect of clamped boundary conditions in the shape of a distributed moment along the simply supported edges [6].

$$
\begin{aligned}
& W_{1}=\frac{4 P a^{4}}{\pi^{5} D} \sum_{m=1,3,5, \ldots}^{\infty} \frac{(-1)^{(m-1) / 2}}{m^{5}}\left(1-\frac{\alpha_{m} \tanh \alpha_{m}+2}{2 \cosh \alpha_{m}} \cosh \frac{m \pi y}{a}\right. \\
& \left.+\frac{1}{2 \cosh \alpha_{m}} \frac{m \pi y}{a} \sinh \frac{m \pi y}{a}\right) \cos \frac{m \pi x}{a}
\end{aligned}
$$

And

$$
\begin{aligned}
& W_{2}=\frac{-a^{2}}{2 \pi^{2} D} \sum_{m=1,3, \ldots}^{\infty} E_{m} \frac{(-1)^{(m-1) / 2} \cos \frac{m \pi x}{a}}{m^{2} \cosh \alpha_{m}} \\
& \left(\frac{m \pi y}{a} \sinh \frac{m \pi y}{a}-\alpha_{m} \tanh \alpha_{m} \cosh \frac{m \pi y}{a}\right)- \\
& \frac{b^{2}}{2 \pi^{2} D} \sum_{m=1,3, \ldots}^{\infty} F_{m} \frac{(-1)^{(m-1) / 2} \cos \frac{m \pi y}{b}}{m^{2} \cosh \beta_{m}} . \\
& \left(\frac{m \pi x}{b} \sinh \frac{m \pi x}{b}-\beta_{m} \tanh \beta_{m} \cosh \frac{m \pi x}{b}\right)
\end{aligned}
$$

where,

$\alpha_{m}=\frac{m \pi b}{2 a}$

And

$\beta_{m}=\frac{m \pi a}{2 b}$

where, $a, b$ are the dimensions of the sensor diaphragm. For calculating the constants $E_{m}$ and $F_{m}$, boundary conditions for the edges $y= \pm b / 2, x= \pm a / 2$ are applied [6]: 
$\left(\frac{\partial w}{\partial y}\right)_{y= \pm b / 2}=\left(\frac{\partial w_{1}}{\partial y}\right)_{y= \pm b / 2}+\left(\frac{\partial w_{2}}{\partial y}\right)_{y= \pm b / 2}=0$

And

$\left(\frac{\partial W}{\partial x}\right)_{x= \pm a / 2}=\left(\frac{\partial w_{1}}{\partial x}\right)_{x= \pm a / 2}+\left(\frac{\partial w_{2}}{\partial x}\right)_{x= \pm a / 2}=0$

By applying the above boundary conditions, we obtain a system for calculating the coefficients $E_{i}$ and $F_{i}$ as follows [6]:

$$
\begin{aligned}
& \frac{4 P a^{2}}{\pi^{3} i^{4}}\left(\frac{\alpha_{i}}{\cosh ^{2} \alpha_{i}}-\tanh \alpha_{i}\right)-\frac{E_{i}}{i}\left(\tanh \alpha_{i}+\frac{\alpha_{i}}{\cosh ^{2} \alpha_{i}}\right) \\
& -\frac{8 i a}{\pi b} \sum_{m=1,3, \ldots}^{\infty} \frac{F_{m}}{m^{3}} \frac{1}{\left(\frac{a^{2}}{b^{2}}+\frac{i^{2}}{m^{2}}\right)^{2}}=0
\end{aligned}
$$

where,

$$
\alpha_{i}=\frac{i \pi b}{2 a}
$$

A similar equation is obtained from Equation (10). The constants $E_{1}, E_{3} \ldots F_{1}, F_{3} \ldots$ can be determined from these two equations by method of successive approximations [6]. The capacitance between two parallel electrodes can be expressed as

$$
C=\varepsilon_{0} \varepsilon_{r} \frac{A}{d}
$$

where, $\varepsilon_{0}, \varepsilon_{\mathrm{r}}, A$ and $d$ are permittivity of free space $\left(8.854 \times 10^{-14} \mathrm{~F} / \mathrm{cm}\right)$, relative dielectric constant of material between the plates (which is unity for air), effective electrode area and gap between the plates, respectively. From this relationship, increasing or decreasing the spacing between the two plates would result in a change in capacitance. The following equation will determine the capacitance changes in a parallel plate capacitor when the electrode is deflected due to an external force [5].

$$
C=\iint_{A} \frac{\varepsilon_{0}}{d-W(x, y)}
$$

where, $d$ is the gap height and $w(x, y)$ is the deflection of the diaphragm. For deflections which are small compared with $h$, the following equation will determine the small signal pressure sensitivity $[8,9]$.

$$
S=\frac{\Delta C}{C_{0} P}
$$

where, $\Delta C$ is the capacitance change and $P$ is related to uniform external pressure.

\section{FINITE ELEMENT ANALYSIS (FEA) OF PRESSURE SENSOR}

The structure of the sensor with clamped diaphragm consists of Pyrex glass back plate, $\mathrm{p}++\mathrm{si}$ diaphragm, and the gold backplate electrode (see Figure 2). In our design, the thickness of the $0.55 \mathrm{~mm} \times 0.55 \mathrm{~mm}$ membrane is $4 \mu \mathrm{m}$, and the height of the air gap is 1.5 $\mu \mathrm{m}$. The Young's modulus and Poisson's ratio of $\mathrm{p}++$ silicon are assumed to be $160 \times 10^{9} \mathrm{~Pa}$ and 0.05 , respectively [10].

FEA software is used for simulating and analyzing the behaviors of MEMS capacitive biosensor to optimize the design and improve the performance of the device. The objectives of analysis are first, to verify the diaphragm displacement due to the mechanically applied pressure between the diaphragm and the back plate. Second, the deflection and capacitance between the diaphragm and the back plate was confirmed. The analysis options are nonlinear analysis, accuracy of convergence is calculated as $0.001 \mu \mathrm{m}$, and a maximum mesh size of is $2.4 \%$ is estimated for $\mathrm{X}-\mathrm{Y}$ dimension.

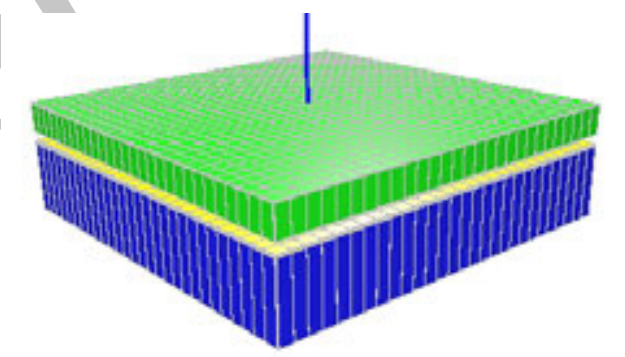

Figure 2. Structure of sensor with clamped diaphragm

\section{RESULTS AND DISCUSSION}

According to Equations (4-12), deflection of a plate with boundary conditions stated in Equation (1) can be calculated. The deformation in the $Z$ axis of the diaphragm with a thickness of $4 \mu \mathrm{m}$ at an applied pressure of $30 \mathrm{mmHg}(4 \mathrm{kPa})$ is investigated analytically and compared with simulation result. Figure 3 shows the analytically calculated deflection with maple software. As can be seen from Figure 3, maximum deflection is occurred at the central point of the plate that is $0.59 \mu \mathrm{m}$.

Figure 4 shows the maximum central deflection of the clamped $\mathrm{p}++$ si diaphragm with FEA software. The result shows the maximum central deflection is 0.535 $\mu \mathrm{m}$ under same situation. As can be seen from the results, theoretical results shows good agreement with finite element analysis. 


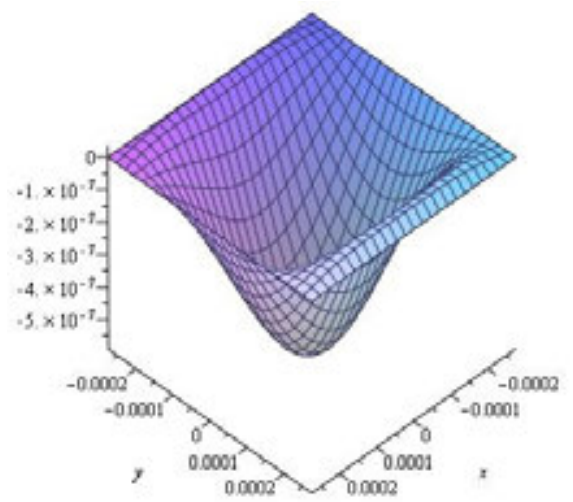

Figure 3. Deflection of a uniformly loaded square plate calculated with maple
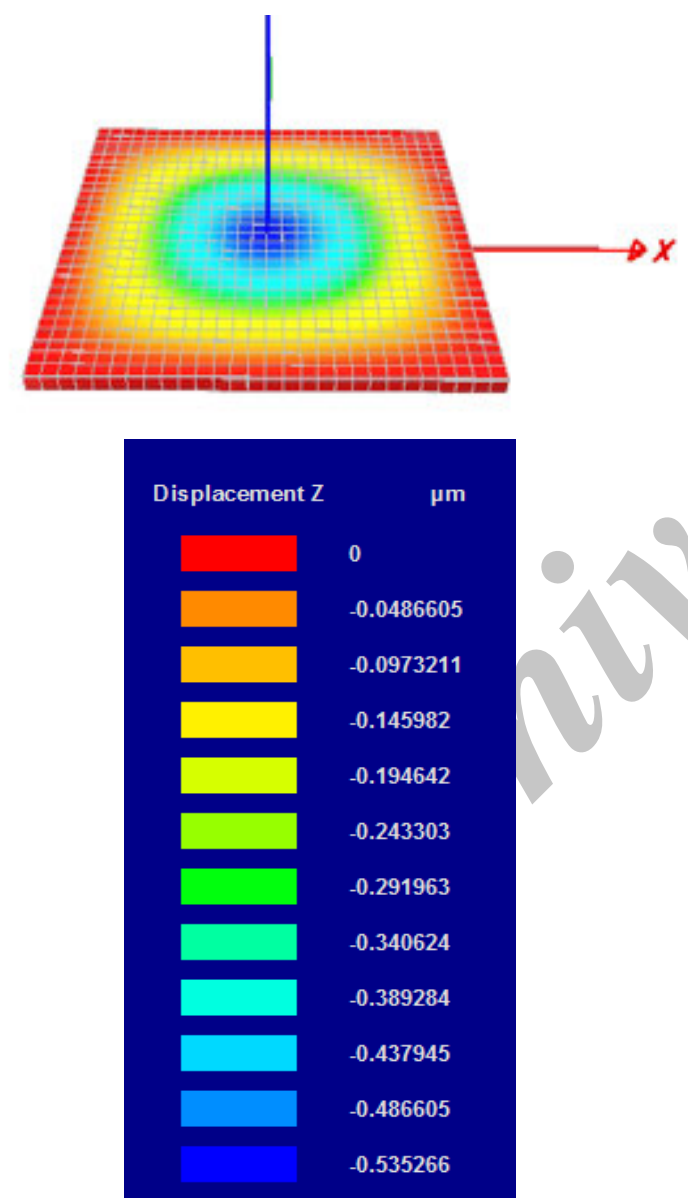

Figure 4. Diaphragm deformation on the $\mathrm{Z}$ axis of the clamped diaphragm

Figure 5 shows the calculated and simulated deflection- pressure curves of a square diaphragm. As can be seen from Figure 5, the central deflection is increased when the applied pressure is increased. From $\left(S_{m}=\frac{d W_{c}}{d P}\right)$ the slope of the curves shows the mechanical sensitivity of the diaphragm. According to Figure 5, the mechanical sensitivity of $1.3 \times 10^{-10} \mathrm{~m} / \mathrm{Pa}$ and $1.45 \times 10^{-10} \mathrm{~m} / \mathrm{Pa}$ have been achieved from simulated and analytical results respectively. It can be seen that the simulation curve is found to be very close to the theoretical curve.

Figure 6 shows the calculated and simulated relation between capacitance and pressure for clamped pressure sensor. From the calculated curve, it can be seen that the initial capacitance of clamped diaphragm is about 1.785 $\mathrm{pF}$ while the initial capacitance of simulated structure is $1.81 \mathrm{pF}$. As pressure applies from $0 \mathrm{mmHg}$ to 60 $\mathrm{mmHg}$, the total variation of capacitance are $0.87 \mathrm{pF}$ and $0.727 \mathrm{pF}$ for calculated and simulated curves, respectively. According to Figure 6, the results yield a sensitivity of $5.02 \times 10^{-5} \mathrm{1} / \mathrm{Pa}$ from simulated structure and $6.09 \times 10^{-5} 1 / \mathrm{Pa}$ from analytical curve. It is clear from Figures 5 and 6 that in higher pressures (more than $40 \mathrm{mmHg}$ ) the difference between simulation and analytical results will be increased. This phenomenon happens because of approximation used for solving very complex mathematical equations of displacement and capacitance.

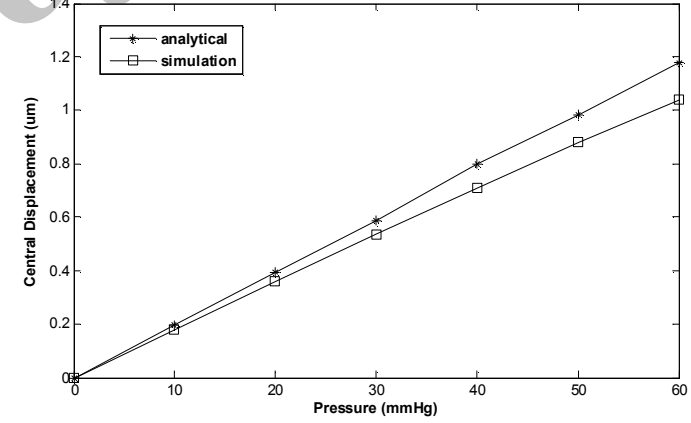

Figure 5. Central displacement versus pressure for the clamped pressure sensor

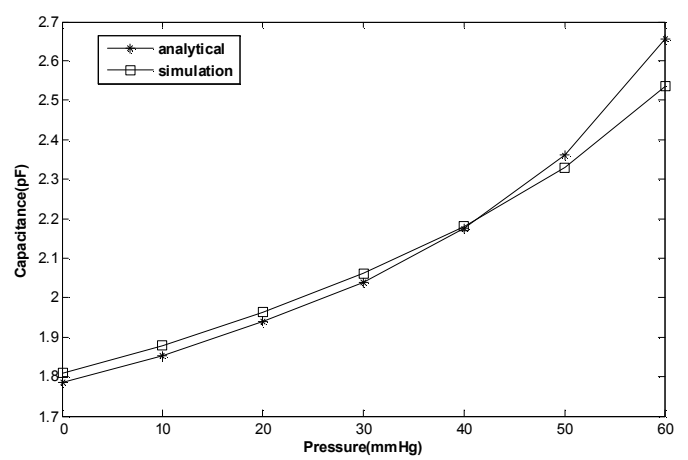

Figure 6. Capacitance versus pressure for the clamped pressure sensor 


\section{CONCLUSION}

In this paper analytical analysis of capacitive pressure sensor with clamped diaphragm is presented and calculated with maple software. The central deflection and capacitance values under the influence of a uniform external pressure are calculated. Furthermore, the calculated results are compared with the simulation using FEA method. Analytical result yields a mechanical sensitivity of $1.45 \times 10^{-10} \mathrm{~m} / \mathrm{Pa}$ and FEA result yields a mechanical sensitivity of $1.3 \times 10^{-10} \mathrm{~m} / \mathrm{Pa}$ for sensor with clamped diaphragm. In addition, the results yield a sensor sensitivity of $5.02 \times 10^{-5} 1 / \mathrm{Pa}$ from simulated structure and $6.09 \times 10^{-5} 1 / \mathrm{Pa}$ from analytical curve. Comparison of the results shows good agreement between theoretical and simulation results. Analysis of structure behavior provides better realization to design high sensitivity pressure sensors.

\section{REFERENCES}

1. Zhou, M. X., Huang, Q. A., Qin, M. and Zhou, W., "A novel capacitive pressure sensor based on sandwich structures", Microelectromechanical Systems, Journal of, Vol. 14, No. 6 , (2005), 1272-1282.
2. Zhang, Y., Howver, R., Gogoi, B. and Yazdi, N., "A highsensitive ultra-thin MEMS capacitive pressure sensor", in SolidState Sensors, Actuators and Microsystems Conference (TRANSDUCERS), 16th International, IEEE, (2011), 112-115.

3. Rahman, M. M. and Chowdhury, S., "Square Diaphragm CMUT Capacitance Calculation Using a New Deflection Shape Function", Journal of Sensors, Vol. 2011, (2011).

4. Ganji, B. A. and Majlis, B. Y., "Fabrication and Characterization of a New MEMS Capacitive Microphone using Perforated Diaphragm", International journal of Engineering, Vol. 22, No. 2, (2009), 153-160.

5. Damghanian, M., Design and Fabrication of a MEMS Tactile Pressure Sensor Array for Fingerprint Imaging, in University of Kebangsaan, Bangi, Malaysia, (2009).

6. Timoshenko, S., Woinowsky-Krieger, S. and Woinowsky, S., "Theory of plates and shells, McGraw-hill New York, Vol. 2, (1959).

7. Di Giovanni, M., "Flat and corrugated diaphragm design handbook, New York, CRC, Marcel Dekker Inc, (1982).

8. Goodall, G. A., "Design of an Implantable Micro-scale Pressure Sensor for Managing Glaucoma", Michigan State University. Department of Mechanical Engineering, (2004).

9. Gu, Y., "Microfabrication of an intraocular pressure sensor, in Electrical and Computer Engineering", Michigan State University, (2006)

10. Senturia, S. D., "Microsystem design. 2001", Boston: Kluwer Academic. Harutyunyan, AR et al., Preferential growth of single-walled carbon nanotubes with metallic conductivity. Science, Vol. 326, No. 5949, (2009), 116-120. 


\title{
Analytical Analysis of Capacitive Pressure Sensor with Clamped RESEARCH Diaphragm
}

\author{
M. Shahiri Tabarestania, B. Azizollah Ganji b \\ a Department of Electrical Engineering, Islamic Azad University, Central Tehran Branch, Tehran, Iran \\ ${ }^{b}$ Electrical Engineering Department, Babol University of Technology, Babol, Iran
}

$P A P E R$ IN FO

Paper history:

Received 24 May 2012

Received in revised form 23 July 2012

Accepted 15 Novemberr 2012

Keywords:

Capacitance

Deflection

Capacitive Pressure Sensor

Diaphragm Deflection
جكيده

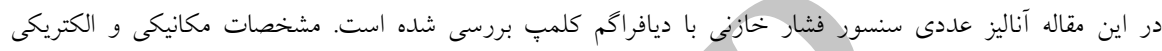

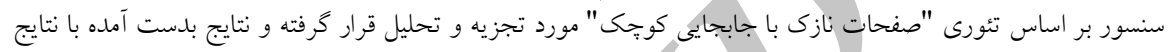

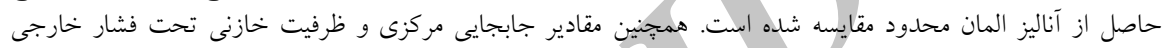

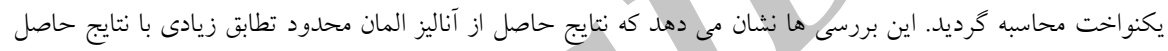

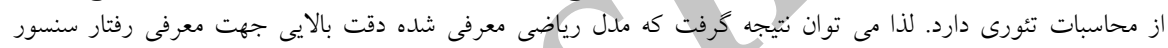
فشار خواهد داشت مخات تئت

doi: 10.5829/idosi.ije.2013.26.03c.09 


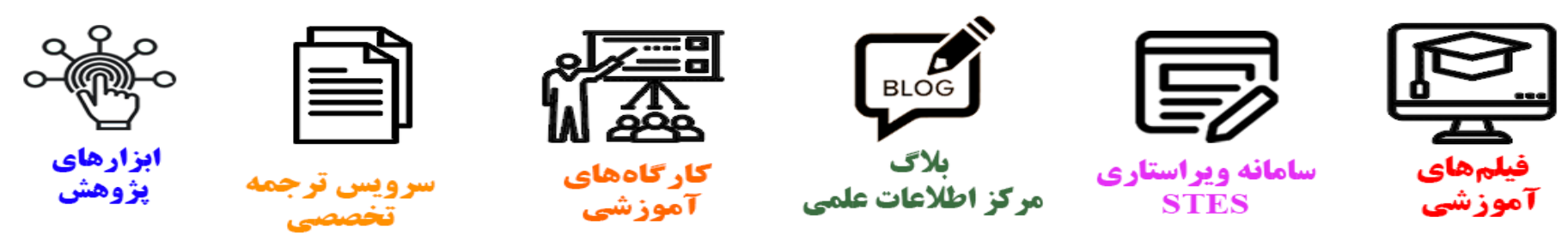

\section{(c)}

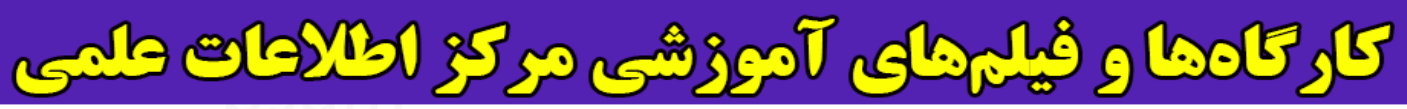
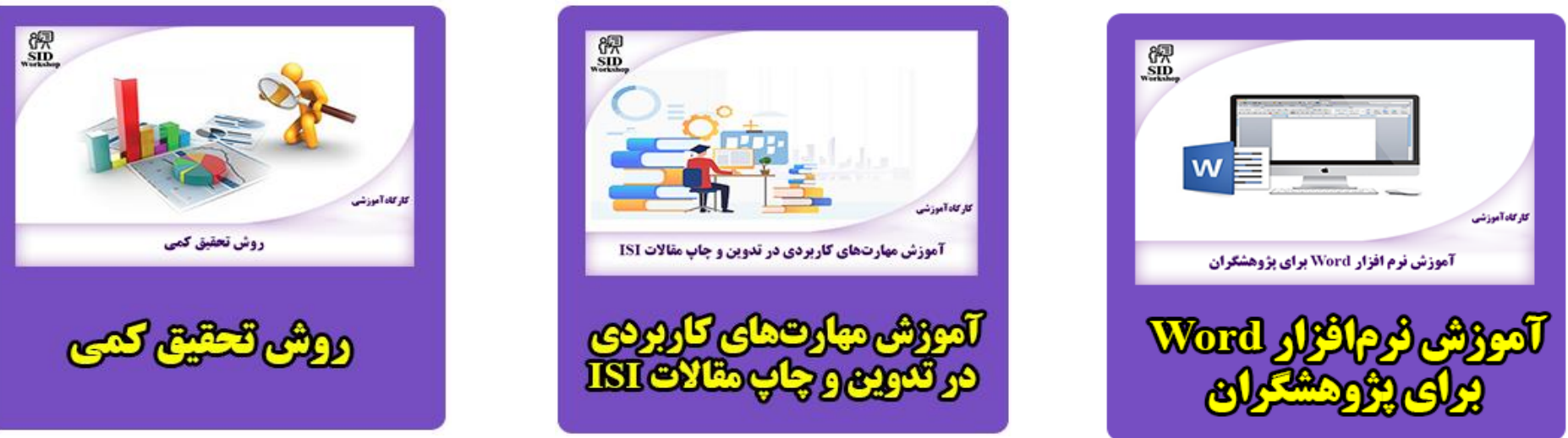